\title{
Two-spin entanglement induced by electron scattering in nanostructures
}

\author{
Gian Luca Giorgi 1,2 , and Ferdinando de Pasquale ${ }^{2,1}$ \\ ${ }^{1}$ CNR-INFM Center for Statistical Mechanics and Complexity \\ ${ }^{2}$ Dipartimento di Fisica, Università di Roma La Sapienza, Piazzale A. Moro 2, 00185 Roma, Italy
}

\begin{abstract}
We present a model where two magnetic impurities in a discrete tight-binding ring become entangled because of scattering processes associated to the injection of a conduction electron. We introduce a weak-coupling approximation that allows us to solve the problem in a analytical way and compare the theory with the exact numerical results. We obtain the generation of entanglement both in a deterministic way and in a probabilistic one. The first case is intrinsically related to the structure of the two-impurity reduced density matrix, while the second one occurs when a projection on the electron state is performed.

PACS numbers: 03.67.Mn, 73.63.-b
\end{abstract}

\section{INTRODUCTION}

The generation of entanglement in mesoscopic structures is considered as a fundamental resource for the implementation of solid-state quantum information processing devices ${ }^{1}$. The first proposals for spin-based quantum computation concern direct interaction between qubits 2 . Further, different schemes for mesoscopic structures have been suggested that create separated streams of entangled particles $3 \cdot 4,5 \cdot 6,7.8$.

Recently, Costa et al.9 have examined the possibility of entangling two spatially separated stationary spins by means of electron scattering. In this case the generation of entanglement would require lower external control. After considering a toy model concerning a ballistic electron interacting in succession with two distant spins, Costa et al. face the more realistic problem of how two magnetic impurities embedded in a solid become entangled because of the injection of a conduction electron, which is scattered by the impurities according to a $s$ - $d$ Hamiltonian. Since in a normal metal described by a tight-binding model the energy spectrum constitutes a continuous band, the appearance of an imaginary part in the eigenenergies of the system is expected that would limit the coherence time of the entangled state. A way to overcome this instability can be represented by the introduction of an artificial discrete system, i.e. a ring of $N$-coupled quantum dots (QDs). During the last decade, electronic transport properties through quantum dots have been widely considered both experimentally and theoretically ${ }^{10}$. Because of the progress of nanotechnology, it is possible to fabricate various structures of coupled QDs smaller than the electron coherence length.

If the interaction term between the electron spin and the impurities is much less than the energy separation between consecutive eigenvalues, in a finite-size system such as a nanostructure, resonance conditions are reached, and a reduction to a few-body system ${ }^{11}$ can be observed. In that case an oscillatory regime is expected to come out also in the degree of entanglement. Dissipation effects could appear only through the interaction with some external bath (for instance, coupling with phonons).
The aim of this paper is to show how entanglement through electron scattering can be generated efficiently in such nanostructures. Then, we consider a finite tightbinding model, and add two magnetic impurities. By studying the dynamical evolution of the state of a conduction electron injected in the chain, we establish the amount of entanglement between the two spins as a function of time. We find that entanglement can be generated in a deterministic way as well as in a probabilistic one.

The plan of the work is the following. After the Introduction, in Sec. II we define the general Hamiltonian model, getting the equations of motion for the states involved in the evolution. In Sec. IIII we establish the approximation of considering the interaction of the incoming mode only with the other resonant modes, and solve the equation of motion. In Sec. IV] the generation of entanglement is studied by means of two different approaches: the measure of concurrence associated to the reduced density matrix obtained by tracing out the electron spin, and the "localizable entanglement" derived from a projective measurement on the electron. In Sec. $\nabla$ we conclude the paper.

\section{MODEL}

We consider a discrete ring structure, described by a standard tight-binding Hamiltonian, where two particular sites (for instance, we label the first site with 0 and the second one with $L$ ) are substituted by magnetic impurities. The $s$ - $d$ Hamiltonian describing the system is

$$
H=H_{0}+\frac{J}{2}\left(\vec{S}_{0} \cdot \vec{\sigma}_{0}+\vec{S}_{L} \cdot \vec{\sigma}_{L}\right)
$$

with $H_{0}=\sum_{k, \sigma} \epsilon_{k} a_{k, \sigma}^{\dagger} a_{k, \sigma}$, where $a_{k, \sigma}^{\dagger}\left(a_{k, \sigma}\right)$ creates (annihilates) one electron with spin $\sigma=\uparrow, \downarrow$ on the mode $k, \epsilon_{k}=-2 w \cos k$ are the eigenvalues of $H$ in the absence of spin interaction ( $w$ is the hopping amplitude between adjacent sites, $k=(2 \pi / N) n, N$ is the total number of sites, and $n$ is an integer running from $-N / 2$ to $[(N / 2)-1]), J$ is the coupling constant between the 


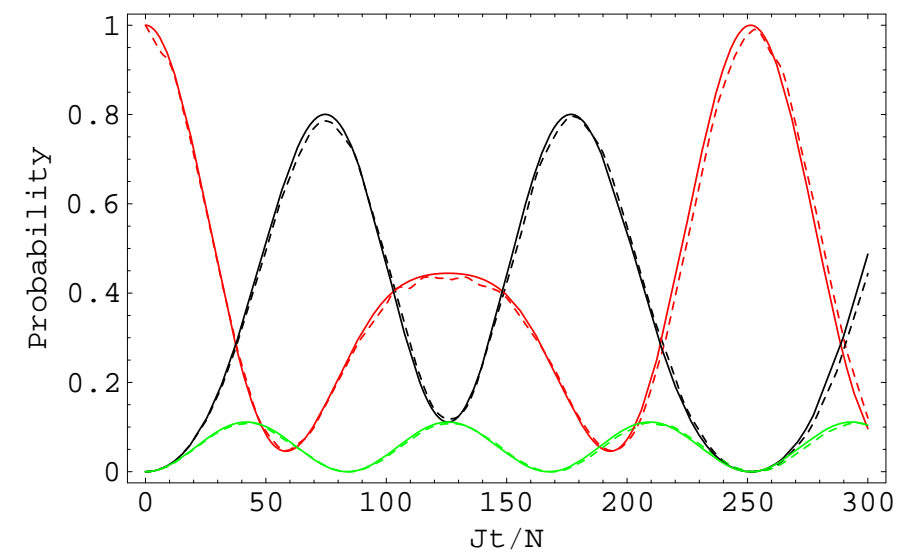

FIG. 1: Plot of the probabilities of the states involved in the evolution as a function of time. Solid lines correspond to theoretical predictions, while dashed lines show numerical (exact) evolution. Red lines concern the state $|\uparrow \uparrow\rangle\left|\downarrow_{k}\right\rangle$, black lines are related to $|\uparrow \uparrow\rangle\left|\downarrow_{-k}\right\rangle$, while green lines regard $|\downarrow \uparrow\rangle\left|\uparrow_{k}\right\rangle$. The probabilities associated to $|\downarrow \uparrow\rangle|\uparrow-k\rangle,|\uparrow \downarrow\rangle\left|\uparrow_{k}\right\rangle$, and $|\uparrow \downarrow\rangle|\uparrow-k\rangle$ are not plotted, being practically indistinguishable from that of $|\downarrow \uparrow\rangle\left|\uparrow_{k}\right\rangle$. The system parameters are the following: $w=1$ is chosen as unit of energy; the number of sites is $N=16$; the scattering amplitude is $J=0.2$, while the distance between the spins is $L=4$.

impurity spins $\vec{S}_{0}$ and $\vec{S}_{L}$ and the electron spins $\vec{\sigma}_{0}$ and $\vec{\sigma}_{L}$, whose operators are defined as

$$
\begin{aligned}
\sigma_{l}^{x} & =a_{l, \uparrow}^{\dagger} a_{l, \downarrow}+a_{l, \downarrow}^{\dagger} a_{l, \uparrow} \\
& =\frac{1}{N} \sum_{q, q^{\prime}}\left(a_{q, \uparrow}^{\dagger} a_{q^{\prime}, \downarrow}+a_{q, \downarrow}^{\dagger} a_{q^{\prime}, \uparrow}\right) e^{i\left(q-q^{\prime}\right) l}, \\
\sigma_{l}^{y} & =-i\left(a_{l, \uparrow}^{\dagger} a_{l, \downarrow}-a_{l, \downarrow}^{\dagger} a_{l, \uparrow}\right) \\
& =\frac{-i}{N} \sum_{q, q^{\prime}}\left(a_{q, \uparrow}^{\dagger} a_{q^{\prime}, \downarrow}-a_{q, \downarrow}^{\dagger} a_{q^{\prime}, \uparrow}\right) e^{i\left(q-q^{\prime}\right) l}, \\
\sigma_{l}^{z} & =a_{l, \uparrow}^{\dagger} a_{l, \uparrow}-a_{l, \downarrow}^{\dagger} a_{l, \downarrow} \\
& =\frac{1}{N} \sum_{q, q^{\prime}}\left(a_{q, \uparrow}^{\dagger} a_{q^{\prime}, \uparrow}-a_{q, \downarrow}^{\dagger} a_{q^{\prime}, \downarrow}\right) e^{i\left(q-q^{\prime}\right) l} .
\end{aligned}
$$

Let us consider the impurities initially with spin up, and the introduction of one excess electron in the state $\left|\downarrow_{k}\right\rangle$. Then, differently from the treatment given in Ref. 9 , we look at entanglement generation from a dynamical point of view, i.e., we analyze the evolution in the time domain of the state $|\uparrow \uparrow\rangle\left|\downarrow_{k}\right\rangle$ to show explicitly how coherent effects persist. By introducing the complex Laplace transform the state evolves in

$$
\begin{aligned}
|\uparrow \uparrow\rangle\left|\downarrow_{k}\right\rangle_{\omega}= & \frac{1}{\omega-\epsilon_{k}}\left[|\uparrow \uparrow\rangle\left|\downarrow_{k}\right\rangle+\right. \\
& \frac{J}{N} \sum_{q}\left(|\downarrow \uparrow\rangle\left|\uparrow_{q}\right\rangle_{\omega}+e^{i(q-k) L}|\uparrow \downarrow\rangle\left|\uparrow_{q}\right\rangle_{\omega}\right) \\
& \left.-\frac{J}{2 N} \sum_{q}\left(1+e^{i(q-k) L}\right)|\uparrow \uparrow\rangle\left|\downarrow_{q}\right\rangle_{\omega}\right],
\end{aligned}
$$

where the notation |\rangle refers to a configuration, while |\rangle$_{\omega}$ refers to the evolution of that configuration, and $\hbar=$ 1. The other states involved in the evolution satisfy the following equations:

$$
\begin{aligned}
|\downarrow \uparrow\rangle\left|\uparrow_{k}\right\rangle_{\omega}= & \frac{1}{\omega-\epsilon_{k}}\left[|\downarrow \uparrow\rangle\left|\uparrow_{k}\right\rangle\right. \\
& +\frac{J}{2 N} \sum_{q}\left(-1+e^{i(q-k) L}\right)|\downarrow \uparrow\rangle\left|\uparrow_{q}\right\rangle_{\omega} \\
& \left.+\frac{J}{N} \sum_{q}|\uparrow \uparrow\rangle\left|\downarrow_{q}\right\rangle_{\omega}\right], \\
|\uparrow \downarrow\rangle\left|\uparrow_{k}\right\rangle_{\omega}= & \frac{1}{\omega-\epsilon_{k}}\left[|\uparrow \downarrow\rangle\left|\uparrow_{k}\right\rangle+\right. \\
& \frac{J}{2 N} \sum_{q}\left(1-e^{i(q-k) L}\right)|\downarrow \uparrow\rangle\left|\uparrow_{q}\right\rangle_{\omega} \\
& \left.+\frac{J}{N} \sum_{q} e^{i(q-k) L}|\uparrow \uparrow\rangle\left|\downarrow_{q}\right\rangle_{\omega}\right] .
\end{aligned}
$$

\section{RESONANT COUPLING APPROXIMATION}

The problem is significantly simplified by introducing the following weak-coupling approximation. In the solution of the system derived from Eqs. (5)-(7), it would appear denominators with the structure $\left[\omega-\epsilon_{k}-\left(J^{2} / N^{2}\right) \sum_{q} f(k-q) /\left(\omega-\epsilon_{q}\right)\right]^{-1}$, where $f(k-q)$ is some weight function derived from $\left( \pm 1 \pm e^{i(q-k) L}\right)$. If the scattering amplitude, of the order of $J / N$, is much less than the energy differences appearing in the spectrum of $H_{0}$, which is about $w / N$ near the middle of the band, we keep just the resonant terms, corresponding to $q= \pm k \stackrel{11}{ }$. For instance,

$$
\begin{array}{r}
\frac{1}{\omega-\epsilon_{k}-\frac{J^{2}}{N^{2}} \sum_{q} \frac{\left(1-e^{i(q-k) L}\right)\left(1+e^{i(q-k) L}\right)}{\omega-\epsilon_{q}}} \\
\simeq \frac{1}{\omega-\epsilon_{k}-\frac{J^{2}}{N^{2}} \frac{1-e^{2 i(q-k) L}}{\omega-\epsilon_{k}}} \\
=\frac{\omega-\epsilon_{k}}{\left(\omega-\epsilon_{k}\right)^{2}-\frac{J^{2}}{N^{2}}\left[1-e^{2 i(q-k) L}\right]},
\end{array}
$$

and in the right-hand side of Eqs. (15)-(17) we maintain only states with momentum $k$ or $-k$, that is, the states that were degenerate with the initial one in absence of 


$$
\begin{aligned}
|\uparrow \uparrow\rangle\left|\downarrow_{k}\right\rangle_{\omega}= & \frac{1}{\omega-\epsilon_{k}+\frac{J}{N}}\left[|\uparrow \uparrow\rangle\left|\downarrow_{k}\right\rangle-\frac{J}{2 N}\left(1+e^{-2 i k L}\right)|\uparrow \uparrow\rangle\left|\downarrow_{-k}\right\rangle_{\omega}\right] \\
& +\frac{1}{\omega-\epsilon_{k}+\frac{J}{N}} \frac{J}{N}\left[|\downarrow \uparrow\rangle\left(\left|\uparrow_{k}\right\rangle_{\omega}+|\uparrow-k\rangle_{\omega}\right)+|\uparrow \downarrow\rangle\left(\left|\uparrow_{k}\right\rangle_{\omega}+e^{-2 i k L}\left|\uparrow-_{-k}\right\rangle_{\omega}\right)\right], \\
|\downarrow \uparrow\rangle\left|\uparrow_{k}\right\rangle_{\omega}= & \frac{1}{\omega-\epsilon_{k}}\left[|\downarrow \uparrow\rangle|\uparrow k\rangle+\frac{J}{2 N}\left(-1+e^{-2 i k L}\right)|\downarrow \uparrow\rangle|\uparrow-k\rangle_{\omega}+\frac{J}{N}|\uparrow \uparrow\rangle\left(\left|\downarrow_{k}\right\rangle_{\omega}+|\downarrow-k\rangle_{\omega}\right)\right], \\
|\uparrow \downarrow\rangle\left|\uparrow_{k}\right\rangle_{\omega}= & \frac{1}{\omega-\epsilon_{k}}\left[|\uparrow \downarrow\rangle\left|\uparrow_{k}\right\rangle+\frac{J}{2 N}\left(1-e^{-2 i k L}\right)|\uparrow \downarrow\rangle|\uparrow-k\rangle_{\omega}+\frac{J}{N}|\uparrow \uparrow\rangle\left(\left|\downarrow_{k}\right\rangle_{\omega}+e^{-2 i k L}\left|\downarrow_{-k}\right\rangle_{\omega}\right)\right] .
\end{aligned}
$$

Together with these equations we must consider also those obtained by exchanging $k$ with $-k$.

A further simplification can be introduced by properly choosing the distance between impurities L. For instance, if $k=\pi / 2$ and $L$ is even, $e^{ \pm 2 i k L}=1$. In this case the study of the state evolution greatly simplifies. Since at $t=0$ we had $|\uparrow \uparrow\rangle\left|\downarrow_{k}\right\rangle$, at the time $t$ we obtain

$$
\begin{aligned}
|\uparrow \uparrow\rangle\left|\downarrow_{k}\right\rangle_{t}= & \frac{1}{6}\left(3+e^{-2 i(J / N) t}+2 e^{4 i(J / N) t}\right)|\uparrow \uparrow\rangle\left|\downarrow_{k}\right\rangle \\
& +\frac{1}{6}\left(-3+e^{-2 i(J / N) t}+2 e^{4 i(J / N) t}\right)|\uparrow \uparrow\rangle|\downarrow-k\rangle \\
& +\frac{1}{6} e^{-2 i(J / N) t}\left(1-e^{6 i(J / N) t}\right)(|\downarrow \uparrow\rangle+|\uparrow \downarrow\rangle)\left(\left|\uparrow_{k}\right\rangle+|\uparrow-k\rangle\right) .
\end{aligned}
$$

The correctness of the above approximation is checked by numerical integration of the Hamiltonian evolution. In Fig. 1 we compare the probabilities derived from the coefficients in Eq. (12) with the exact results. The agreement between perturbation theory and numerical results is remarkable.

\section{ENTANGLEMENT MEASURES}

The two-impurity reduced density matrix $\rho$ is obtained by tracing out the electron degree of freedom: in the basis spanned by the states $\{|\uparrow \uparrow\rangle,|\downarrow \uparrow\rangle,|\uparrow \downarrow\rangle,|\downarrow \downarrow\rangle\}$ we have

$$
\rho=\left(\begin{array}{cccc}
1-\frac{4}{9} \sin ^{2} \frac{3 J}{N} t & 0 & 0 & 0 \\
0 & \frac{2}{9} \sin ^{2} \frac{3 J}{N} t & \frac{2}{9} \sin ^{2} \frac{3 J}{N} t & 0 \\
0 & \frac{2}{9} \sin ^{2} \frac{3 J}{N} t & \frac{2}{9} \sin ^{2} \frac{3 J}{N} t & 0 \\
0 & 0 & 0 & 0
\end{array}\right) .
$$

Given $\rho$, we can compute the corresponding degree of entanglement by means of the concurrence $\mathcal{C}^{12}$. The concurrence between two qubits is defined to be $\mathcal{C}=$ $\max \left\{\lambda_{1}-\lambda_{2}-\lambda_{3}-\lambda_{4}, 0\right\}$, where $\lambda_{r}$ is the square root of the $r$ th eigenvalue of $R=\rho \tilde{\rho}$ in descending order. The matrix $\tilde{\rho}$ is defined as $\tilde{\rho}=\left(\sigma_{y} \otimes \sigma_{y}\right) \rho^{*}\left(\sigma_{y} \otimes \sigma_{y}\right)$, where $\rho^{*}$ is the complex conjugate of $\rho$. Since the eignevalues of $R$ are $\left\{0,0,0,(16 / 81) \sin ^{4}(3 J t / N)\right\}$, the corresponding $\lambda_{r}$ are $\left\{0,0,0,(4 / 9) \sin ^{2}(3 J t / N)\right\}$. So we have $\mathcal{C}=4 / 9 \sin ^{2}(3 J t / N)$. In Fig. 20 we report the numerical value of $\mathcal{C}$ as a function of time and compare it with the analytical expression. In this way we have calculated the amount of entanglement arising spontaneously from scattering processes. Actually, there is a different strategy from which a higher degree of entanglement, the socalled localizable entanglement ${ }^{13.14}$, could be extracted. It consists in a projective measurement performed on the electron degree of freedom. If we project $|\uparrow \uparrow\rangle\left|\downarrow_{k}\right\rangle_{t}$ onto $\left(\left|\uparrow_{k}\right\rangle+\left|\uparrow_{-k}\right\rangle\right) / \sqrt{2}$ we get the state

$$
\frac{\left\langle\uparrow_{k}\right|+\langle\uparrow-k|}{\sqrt{2}}|\uparrow \uparrow\rangle\left|\downarrow_{k}\right\rangle_{t}=\frac{e^{-2 i(J / N) t}-e^{4 i(J / N) t}}{3} \frac{|\downarrow \uparrow\rangle+|\uparrow \downarrow\rangle}{\sqrt{2}} .
$$

As a result of the projective measurement, a maximally entangled state appears for all the times. However, the probability $P$ of actually finding $\left(\left|\uparrow_{k}\right\rangle+\left|\uparrow_{-k}\right\rangle\right) / \sqrt{2}$ is dif- 


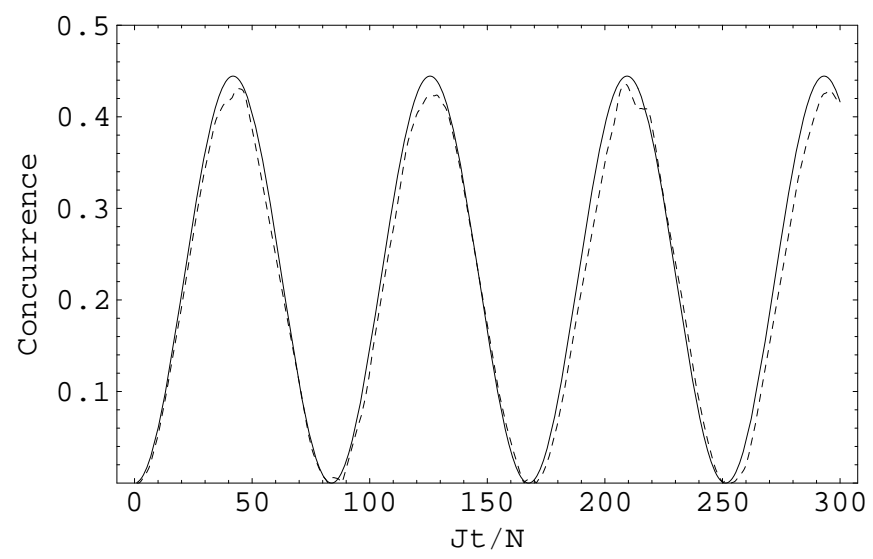

FIG. 2: Concurrence as a function of time. The solid line is derived from the theoretical model described in the text, while the dashed line corresponds to the numerical calculation. The system parameters are the same as those defined in the caption of Fig. [

ferent from one;

$$
P=\left|\frac{1}{3}\left(e^{-2 i(J / N) t}-e^{4 i(J / N) t}\right)\right|^{2}=\frac{4}{9} \sin ^{2} \frac{3 J}{N} t .
$$

That is, the process is probabilistic instead of deterministic. We obtain a success probability that evolves in time with the same law of $\mathcal{C}$. The two results have the following interpretation. In the first case the two spins are spontaneously entangled by electron scattering. The amount of entanglement is obviously related to the probability of finding the component $\left(|\downarrow \uparrow\rangle\left|\uparrow_{k}\right\rangle+|\downarrow \uparrow\rangle\left|\uparrow{ }_{-k}\right\rangle+|\uparrow \downarrow\rangle\left|\uparrow_{k}\right\rangle+|\uparrow \downarrow\rangle\left|\uparrow{ }_{k}\right\rangle\right)$. In the physical procedure related to the projective measurement, we consider only this component. The same time evolution is then associated to two different kinds of processes, the first one being deterministic and the second one being probabilistic. In fact, without any kind of projection, we have a limited degree of entanglement. By projecting on the electron state we can reach $\mathcal{C}=1$, but the price to pay consists in a limited success probability of the projection. The best strategy to adopt will depend on the specific application.

\section{CONCLUSIONS}

In this paper we have discussed the problem of entangling two distant spins embedded in a solid-state environment through the interaction with a conduction electron. We have described explicitly what happens when a finitesize system is considered. By applying a weak-coupling approximation, that is, by neglecting nonresonant scattering states, we have solved analytically the evolution in time of the state associated to an incoming conduction electron. In fact, the existence of discrete levels and the weakness of the coupling make possible a resonance between the scattered states and one level of the energy band. A comparison between theoretical results and exact numerical results has been presented, showing the accuracy of the weak-coupling approximation. As a result of the evolution, two-spin entanglement appears. We have analyzed the emergence of entanglement merely from evolution through the reduced density matrix, and the role of projection on the electron spin state. In conclusion, magnetic scattering in a discrete system has been shown to create entanglement both in a deterministic and in a probabilistic way.
* Electronic address: gianluca.giorgi@roma1.infn.it

1 M. A. Nielsen and I. L. Chuang, Quantum Computation and Quantum Information (Cambridge Univ. Press, Cambridge, 2000).

2 D. Loss and D. P. DiVincenzo, Phys. Rev. A 57, 120 (1998).

3 A. V. Lebedev, G. Blatter, C. W. J. Beenakker, and G. B. Lesovik, Phys. Rev. B 69, 235312 (2004).

4 C. Bena, S. Vishveshwara, L. Balents, and M.P.A. Fisher, Phys. Rev. Lett. 89, 037901 (2002).

5 D. Loss and E.V. Sukhorukov, Phys. Rev. Lett. 84, 1035 (2000).

${ }^{6}$ G. Burkard, D. Loss, and E.V. Sukhorukov, Phys. Rev. B 61, R16303 (2000);

7 P. Recher, E.V. Sukhorukov, and D. Loss, Phys. Rev. B 63, 165314 (2001).
8 P. Samuelsson, E.V. Sukhorukov, and M. Büttiker, Phys. Rev. Lett. 91, 157002 (2003).

9 A.T. Costa, Jr., S. Bose, and Y. Omar, Phys. Rev. Lett. 96, 230501 (2003).

10 W. G. van der Wiel, S. De Franceschi, J. M. Elzerman, T. Fujisawa, S. Tarucha, and L. P. Kouwenhoven, Rev. Mod. Phys. 75, 1 (2003).

11 S. Paganelli, F. de Pasquale, and G. L. Giorgi, Phys. Rev. A 74, 012316 (2006).

12 W. K. Wootters, Phys. Rev. Lett. 80, 2245 (1998).

13 M. Popp, F. Verstraete, M. A. Martín-Delgado, and J. I. Cirac Phys. Rev. A 71, 042306 (2005).

14 F. Verstraete, M. Popp, and J. I. Cirac Phys. Rev. Lett. 92, 027901 (2004). 